\title{
Certified Reference Material recipe challenge
}

\author{
Catherine A. Rimmer • Melissa M. Phillips
}

(C) Springer-Verlag Berlin Heidelberg 2013

We would like to invite you to participate in the Analytical Challenge, a series of puzzles to entertain and challenge our readers. This special feature of "Analytical and Bioanalytical Chemistry" has established itself as a truly unique quiz series, with a new scientific puzzle published every other month. Readers can access the complete collection of published problems with their solutions on the ABC homepage at http://www.springer.com/abc. Test your knowledge and tease your wits in diverse areas of analytical and bioanalytical chemistry by viewing this collection.

In the present challenge, Certified Reference Materials are the topic. And please note that there is a prize to be won (a Springer book of your choice up to a value of $€ 100)$. Please read on...

\section{Meet the Certified Reference Material recipe challenge}

Chemistry, biochemistry, and cooking go hand in hand. For example, yeast in the presence of sugar, moisture, and gentle heat will release carbon dioxide and alcohol. When yeast is used in bread making, the carbon dioxide causes the dough to rise and the alcohol contributes to the flavor of the bread. There are also factors that inhibit the reaction. If, for example, the temperature is too low during the proofing and rising steps, the yeast will

C. A. Rimmer $(\triangle) \cdot$ M. M. Phillips

Analytical Chemistry Division, National Institute of Standards and Technology, 100 Bureau Drive, Stop 8392,

Gaithersburg, MD 20899-8392, USA

e-mail: catherine.rimmer@nist.gov remain dormant. If the temperature is too high, the yeast will be killed. At either extreme, the dough fails to rise. Salt can be used to slow the formation of carbon dioxide, whereas sugar can speed it up. The Maillard reaction of denatured proteins with sugars also plays an important role in the aroma and flavor of foods from bread to beef. With a little chemical knowledge, the reaction can be used to maximize the flavor of cooked foods.

It is no secret that the quality of the ingredients used in cooking plays a significant role in the flavor of the food as well as the nutritional benefits. But how does a laboratory demonstrate the quality of a food? The measurement of vitamins, elements, fatty acids, contaminants, and volatile flavor components in complex food matrices presents significant analytical challenges.

The issue of Analytical and Bioanalytical Chemistry in which this challenge is published is related to the analysis of foods and dietary supplements, an area in which quality is also an important theme. Certified Reference Materials (CRMs) are essential tools for the quantitative determination of analytes in foods and dietary supplements. Sample preparation and complete analyte extraction are often the most challenging steps in the analysis; thus, it is essential to have well-matched matrix-based CRMs for use as quality control materials and method development tools. As described by Phillips et al. [1], AOAC International has developed a food triangle with $100 \%$ fat, $100 \%$ protein, and $100 \%$ carbohydrate as the apices. The National Institute of Standards and Technology has worked to fill in the different sectors of the triangle with Standard Reference Materials derived from foods to represent different analytical challenges associated with food analysis. Other governmental national metrology institutes, members of private industry, and nonprofit foundations have created additional CRMs for community use. 


\section{The challenge}

The challenge is to create a recipe using only CRMs. Please be sure to include the list of CRMs with quantities, a cost estimate of the ingredients (how many units of each CRM must be purchased and at what cost), and preparation/cooking instructions. Points will be assigned for:

1. The number of CRMs used (is the dish made exclusively from CRMs, or did you require non-CRM ingredients?)

2. The cost of the recipe (in this case, higher is better)

3. Creativity of the directions

4. Gastronomic appeal

We hope that you enjoy this unique challenge. Bon appétit.

\section{Reference}

1. Phillips MM, Sharpless KE, Wise SA (2013) Anal Bioanal Chem. doi:10.1007/s00216-013-6890-5

We invite our readers to participate in the Analytical Challenge by solving the puzzle above. Please send the correct solution to abc-challenge@springer.comby June 10, 2013. Make sure you enter "Certified Reference Material challenge" in the subject line of your e-mail. The winner will be notified by e-mail and his/her name will be published on the ABC homepage at http://www.springer.com/abc and in the journal (volume 405/issue 22), where readers will find the solution and a short explanation.

The next Analytical Challenge will be published in volume 405/17, July 2013. If you have enjoyed solving this Analytical Challenge you are invited to try the previous puzzles on the ABC homepage. 